\title{
A GENERAL MODEL BASED ON ADDITIVE WEIBULL DISTRIBUTION: WITH APPLICATION
}

\author{
S. M. MOHAMED and A. A. E. AHMED \\ Institute of Statistical Studies \& Research \\ Cairo University \\ Egypt \\ College of Commerce \& Business Administration \\ Hellwan University \\ Egypt \\ e-mail: ahmed63feb@gmail.com
}

\begin{abstract}
This paper introduces a new generalization of the transmuted additive Weibull distribution by Elbatal and Aryal [3], based on a new family of lifetime distribution. We refer to the new distribution as Kumaraswamy new transmuted additive Weibull (Kw-NTAW) distribution. The new model contains more than seventy of lifetime distributions as special cases such as the transmuted additive Weibull, Kumaraswamy-transmuted exponentiated modified Weibull (Kw-TEMW), exponentiated modified Weibull, exponentiated Weibull, exponentiated exponential, transmuted Weibull, Rayleigh, linear failure rate, and exponential distributions, among others. The properties of the new model are discussed. Explicit expressions are derived for the moments. An application to real data set is finally presented for illustration.
\end{abstract}

2010 Mathematics Subject Classification: 47Jxx, 47J10, 47J35.

Keywords and phrases: Kumaraswamy distribution, transmutation, survival function, exponentiated exponential, order statistics, maximum likelihood estimation.

Received November 22, 2015

(ㄷ) 2016 Scientific Advances Publishers 


\section{Introduction}

This paper introduces a generalization of the method introduced by Mansour and Hamed [7] using Kumaraswamy distribution introduced by Cordeiro and de Castro [2]. Due to its importance in accommodating all forms of the hazard rate function as seen from Figure 2 (by changing its parameter values) seems to be an important distribution that can be used, it is known not many lifetime distributions exhibit bathtub hazard rates. Distributions with such failure rates have attracted a considerable attention of researchers in reliability engineering. In software reliability, bathtub shaped failure rate is encountered in firmware, and in embedded software in hardware devices. Firmware plays an important role in functioning of hard drives of large computers, spacecraft and high performance aircraft control systems, advanced weapon systems, safety critical control systems used for monitoring the industrial process in chemical and nuclear plants (Zhang et al. [9]). The upside down bathtub shaped failure rate is used in data of motor bus failures (Mudholkar et al. [8]), for ageing properties in reliability (Gupta et al. [4]; Jiang et al. [5]) and the course of a disease whose mortality reaches a peak after some finite period and then declines gradually.

In this article, we use transmutation map approach suggested by Mansour and Hamed [7] to define a new model. Let $G(x)$ be the cumulative distribution function (cdf) of a non-negative absolutely continuous random variable, $G(x)$ be strictly increasing on its support, and $G(0)=0$ define a new cdf, $F(x)$, out of $G(x)$ as

$$
F(x)=(1+\lambda)[G(x)]^{\delta}-\lambda[G(x)]^{\alpha}, \quad x>0,
$$

where $\alpha, \delta>0$ for $0>\lambda>-1$, and $\alpha>0,(5 \alpha / 4) \geq \delta \geq(\alpha / 2)$ for $0<\lambda<1$. 
Kumaraswamy [6] introduced a two-parameter distribution on $(0,1)$, which will be referred to by "Kw" in the sequel. Its cdf is given by

$$
F(x)=1-\left(1-x^{a}\right)^{b}, \quad x \in(0,1),
$$

where $a>0$ and $b>0$ are shape parameters. The model in (2) compares extremely favorably in terms of simplicity with the beta cdf, that is, the incomplete beta function ratio. The pdf corresponding to (2) is given by,

$$
f(x)=a b\left(1-x^{a}\right)^{b-1}, \quad x \in(0,1) .
$$

The Kw density function has the same basic shape properties of the beta distribution: $a>1$ and $b>1$ (unimodal); $a<1$ and $b<1$ (uniantimodal); $a>1$ and $b \leq 1$ (increasing); $a \leq 1$ and $b>1$ (decreasing); $a=b=1$ (constant).

For an arbitrary baseline cdf, $G(x)$ Cordeiro and de Castro [2] defined the Kw-G distribution by the pdf $f(x)$ and $\operatorname{cdf} F(x)$ as

$$
f(x)=a b \cdot g(x) \cdot G^{a-1}(x)\left[1-G^{a}(x)\right]^{b-1},
$$

and

$$
F(x)=1-\left[1-G^{a}(x)\right]^{b},
$$

respectively, where $g(x)=d G(x) / d x$ and $a$ and $b$ are two extra positive shape parameters. It follows immediately from (5) that the $\mathrm{Kw}-G$ distribution with parent $\operatorname{cdf} G(x)=x$ produces the minimax distribution (2). If $X$ is a random variable with pdf (4), we write $X \sim K w-G(a, b)$, where $a$ and $b$ are additional shape parameters which aim to govern skewness and tail weight of the generated distribution. An attractive feature of this distribution is that the two parameters $a$ and $b$ can afford greater control over the weights in both tails and in its centre. 


\section{Kumaraswamy New Transmuted Additive Weibull Distribution}

In this section, we introduce a new distribution called the Kumaraswamy new transmuted additive Weibull distribution denoted by Kw-NTAW. Then the cumulative distribution function of Kw-NTAW model (for $x>0$ ) denoted by $F(x, \lambda, \theta, \nu, \gamma, \beta, \delta, \alpha, a, b) \equiv F(x)$ becomes

$$
F(x)=1-\left\{1-\left\{(1+\lambda)[I(x, \vartheta)]^{\delta}-\lambda[I(x, \vartheta)]^{\alpha}\right\}^{a}\right\}^{b}
$$

where

$$
I(x, \vartheta)=1-e^{-\left(\theta x^{\nu}+\gamma x^{\beta}\right)}
$$

where as its pdf can be expressed,

$$
\begin{aligned}
f(x)= & \text { a.b. }\left(\theta \nu x^{\nu-1}+\gamma \beta x^{\beta-1}\right) e^{-\left(\theta x^{\nu}+\gamma x^{\beta}\right)}\left[(1+\lambda) \delta[I(x, \vartheta)]^{\delta-1}-\lambda \alpha[I(x, \vartheta)]^{\alpha-1}\right] \\
& \times\left\{(1+\lambda)[I(x, \vartheta)]^{\delta}-\lambda[I(x, \vartheta)]^{\alpha}\right\}^{a-1} \\
& \times\left\{1-\left\{(1+\lambda)[I(x, \vartheta)]^{\delta}-\lambda[I(x, \vartheta)]^{\alpha}\right\}^{\alpha}\right\}^{b-1}
\end{aligned}
$$

where $\beta>0, \nu, \delta>0, \alpha>0, a>0$, and $b>0$ are shape parameters with $\beta<1<\nu$ or $(\nu<1<\beta)$, and $\theta>0$ and $\gamma>0$ are scale parameters and $|\lambda|$ is a transmuted parameter. The random variable $x$ with the density function (7) is said to have a Kumaraswamy new transmuted additive Weibull (Kw-NTAW) distribution.

The flexibility of the Kw-NTAW is explained in Table 1 when their parameters are carefully chosen. 
Table 1. The special cases of the Kw-NTAW distribution

\begin{tabular}{|c|c|c|c|c|c|c|c|c|c|c|}
\hline \multirow{2}{*}{ Distribution } & \multicolumn{9}{|c|}{ Parameters } & \multirow{2}{*}{ Author } \\
\hline & $a$ & $b$ & $\lambda$ & $\delta$ & $\alpha$ & $\theta$ & $v$ & $\gamma$ & $\beta$ & \\
\hline Kw-TEMW & & & & $\alpha / 2$ & & & 1 & & & New \\
\hline Kw-TEAW & & & & & & & & & & New \\
\hline Kw-TELFR & & & & 1 & & & 1 & & 2 & \\
\hline Kw-TEW & & & & 1 & & 0 & 1 & & & New \\
\hline Kw-TER & & & & 1 & & 0 & 1 & & 2 & New \\
\hline Kw-TEE & & & & 1 & & & 1 & 0 & & New \\
\hline Kw-TMW & & & & 1 & 2 & & 1 & & & New \\
\hline Kw-TLFR & & & & 1 & 2 & & 1 & & 2 & New \\
\hline Kw-TW & & & & 1 & 2 & 0 & 1 & & & New \\
\hline Kw-TR & & & & 1 & 2 & 0 & 1 & & 2 & New \\
\hline Kw-TE & & & & 1 & 2 & & 1 & 0 & & New \\
\hline Kw-EMW & & & 0 & $\alpha / 2$ & & & 1 & & & New \\
\hline Kw-ELFR & & & 0 & & & & 1 & & 2 & \\
\hline Kw-EW & & & 0 & $\alpha / 2$ & & 0 & & & & New \\
\hline Kw-ER & & & 0 & $\alpha / 2$ & & 0 & & & 2 & \\
\hline Kw-EE & & & 0 & & & & 1 & 0 & & \\
\hline Kw-MW & & & 0 & 1 & & & 1 & & & New \\
\hline Kw-LFR & & & 0 & 1 & & & 1 & & 2 & \\
\hline Kw-MW & & & 0 & 1 & & 0 & & & & Cordeiro and de Castro [2] \\
\hline Kw-R & & & 0 & 1 & & 0 & & & 2 & \\
\hline $\mathbf{K w}-\mathbf{E}$ & & & 0 & 1 & & & 1 & 0 & & \\
\hline TEAW & 1 & 1 & & $\alpha / 2$ & & & & & & \\
\hline TAW & 1 & 1 & & 1 & 1 & & & & & \\
\hline EAW & 1 & 1 & 0 & & & & & & & \\
\hline AW & 1 & 1 & 0 & 1 & & & & & & \\
\hline ETEMW & & 1 & & $\alpha / 2$ & & & 1 & & & New \\
\hline ETELFR & & 1 & & $\alpha / 2$ & & & 1 & & 2 & New \\
\hline ETEW & & 1 & & $\alpha / 2$ & & 0 & & & & New \\
\hline ETER & & 1 & & $\alpha / 2$ & & 0 & & & 2 & New \\
\hline
\end{tabular}


Table 1. (Continued)

\begin{tabular}{|c|c|c|c|c|c|c|c|c|c|c|}
\hline ETEE & & 1 & & $\alpha / 2$ & & & 1 & 0 & & New \\
\hline ETMW & & 1 & & 1 & 2 & & 1 & & & New \\
\hline ETLFR & & 1 & & 1 & 2 & & 1 & & 2 & New \\
\hline ETW & & 1 & & 1 & 2 & 0 & & & & New \\
\hline ETR & & 1 & & 1 & 2 & 0 & & & 2 & New \\
\hline ETE & & 1 & & 1 & 2 & & 1 & 0 & & New \\
\hline New-EMW & & 1 & 0 & & & & 1 & & & New \\
\hline New-ELFR & & 1 & 0 & & & & 1 & & 2 & New \\
\hline New-EW & & 1 & 0 & & & 0 & & & & New \\
\hline New-ER & & 1 & 0 & & & 0 & & & 2 & New \\
\hline New-EE & & 1 & 0 & & & & 1 & 0 & & New \\
\hline EMW & & 1 & 0 & 1 & & & 1 & & & Elbatal and Aryal [3] \\
\hline EW & & 1 & 0 & 1 & & 0 & & & & Mudholkar and Srivastava [8] \\
\hline EE & & 1 & 0 & 1 & 0 & & 1 & 0 & & Gupta et al. [4] \\
\hline TEMW & 1 & 1 & & $\alpha / 2$ & & & 1 & & & \\
\hline TELFR & 1 & 1 & & $\alpha / 2$ & & & 1 & & 2 & \\
\hline TEW & 1 & 1 & & $\alpha / 2$ & & 0 & & & & \\
\hline TER & 1 & 1 & & $\alpha / 2$ & & 0 & & & 2 & \\
\hline TEE & 1 & 1 & & $\alpha / 2$ & & & 1 & 0 & & \\
\hline TMW & 1 & 1 & & 1 & 2 & & 1 & & & \\
\hline TLFR & 1 & 1 & & 1 & 2 & & 1 & & 2 & \\
\hline TW & 1 & 1 & & 1 & 2 & 0 & & & & \\
\hline TR & 1 & 1 & & 1 & 2 & 0 & & & 2 & \\
\hline TE & 1 & 1 & & 1 & 2 & & 1 & 0 & & \\
\hline ELFR & 1 & 1 & 0 & & & & 1 & & 2 & \\
\hline ER & 1 & 1 & 0 & & & 0 & & & 2 & \\
\hline MW & 1 & 1 & 0 & 1 & & & 1 & & & \\
\hline LFR & 1 & 1 & 0 & & 1 & & 1 & & 2 & \\
\hline $\mathbf{W}$ & 1 & 1 & 0 & 1 & & 0 & & & & \\
\hline $\mathbf{R}$ & 1 & 1 & 0 & & 1 & 0 & & & 2 & \\
\hline $\mathbf{E}$ & 1 & 1 & 0 & 1 & & & 1 & 0 & & \\
\hline
\end{tabular}

Figure 1 illustrates some of the possible shapes of the pdf of the Kw-NTAW distribution for selected values of the parameters $\lambda, \theta, \nu, \gamma, \beta, \delta, \alpha, a$, and $b$, respectively. 

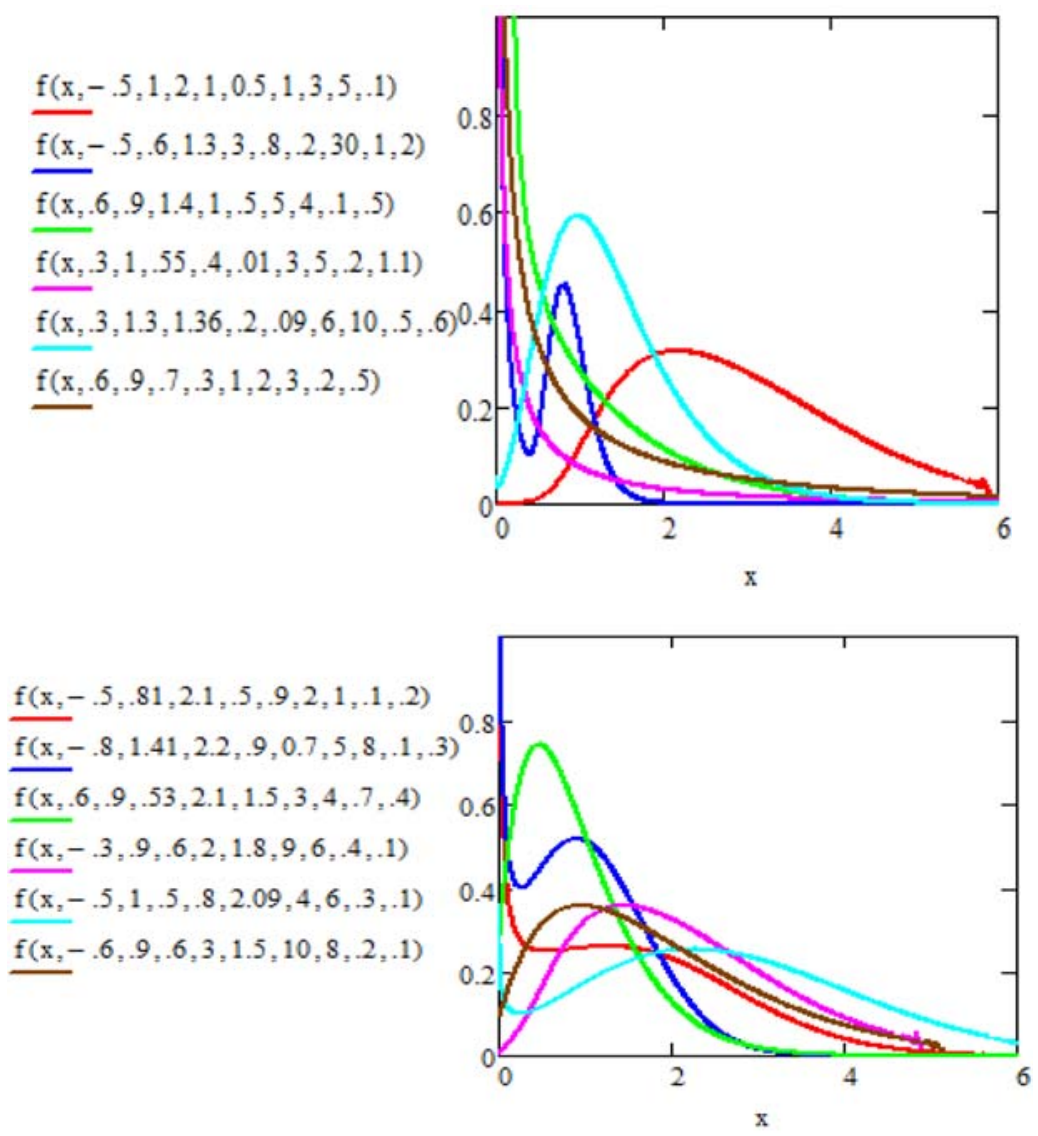

Figure 1. Probability density function of the Kw-NTAW distribution.

\section{Reliability Analysis}

\subsection{Reliability function}

The reliability function of the Kw-NTAW distribution denoted by $\mathrm{R}_{\mathrm{Kw}-\mathrm{NTAW}}(\lambda, \theta, \nu, \gamma, \beta, \delta, \alpha, a, b)$, can be a useful characterization of lifetime data analysis. It can be defined as,

$$
\begin{aligned}
\mathrm{R}_{\mathrm{Kw}-\mathrm{NTAW}}(x, \lambda, \theta, \nu, \gamma, \beta, \delta, \alpha, a, b) & \\
= & 1-\mathrm{F}_{\mathrm{Kw}-\mathrm{NTAW}}(x, \lambda, \theta, \nu, \gamma, \beta, \delta, \alpha, a, b),
\end{aligned}
$$


the survival function of is given by,

$$
\begin{aligned}
& \mathrm{R}_{\mathrm{Kw}-\mathrm{NTAW}}(x, \lambda, \theta, \nu, \gamma, \beta, \delta, \alpha, a, b) \\
&=\left\{1-\left\{(1+\lambda)[I(x, \vartheta)]^{\delta}-\lambda[I(x, \vartheta)]^{\alpha}\right\}^{a}\right\}^{b} .
\end{aligned}
$$

\subsection{Hazard rate function}

The failure rate function is given by

$$
\begin{aligned}
h(x)=a . b .\left(\theta \nu x^{\nu-1}\right. & \left.+\gamma \beta x^{\beta-1}\right) e^{-\left(\theta x^{\nu}+\gamma x^{\beta}\right)}\left[(1+\lambda) \delta[I(x, \vartheta)]^{\delta-1}-\lambda \alpha[I(x, \vartheta)]^{\alpha-1}\right] \\
& \times \frac{\left\{(1+\lambda)[I(x, \vartheta)]^{\delta}-\lambda[I(x, \vartheta)]^{\alpha}\right\}^{\alpha-1}}{1-\left\{(1+\lambda)[I(x, \vartheta)]^{\delta}-\lambda[I(x, \vartheta)]^{\alpha}\right\}^{\alpha}} .
\end{aligned}
$$



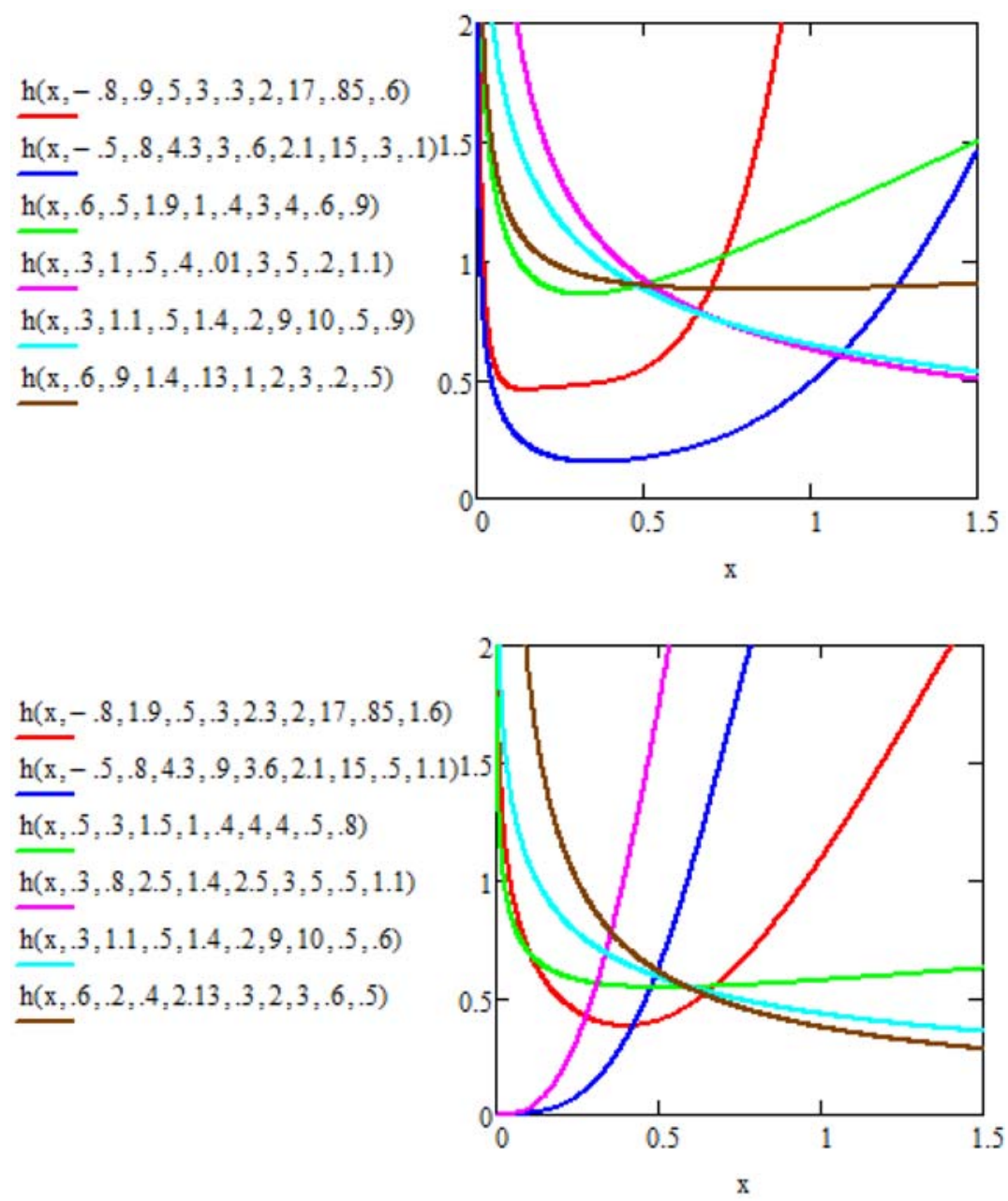

Figure 2. Hazard rate of the Kw-NTAW distribution.

\subsection{Cumulative hazard rate function}

The cumulative hazard function (CHF) of the Kumaraswamy new transmuted additive Weibull distribution, denoted by $\mathrm{H}_{\mathrm{Kw}-\mathrm{NTAW}}(x, \lambda$, $\theta, \nu, \gamma, \beta, \delta, \alpha)$, is defined as 
$\mathrm{H}_{\mathrm{Kw}-\mathrm{NTAW}}(x, \lambda, \theta, \nu, \gamma, \beta, \delta, \alpha, \alpha, b)=\int_{0}^{x} \mathrm{~h}_{\mathrm{Kw}-\mathrm{NTAW}}(x, \lambda, \theta, \nu, \gamma, \beta, \delta, \alpha, \mathrm{a}, b) d x$

$$
=-\ln \mathrm{R}_{\mathrm{Kw}-\mathrm{NTAW}}(x, \lambda, \theta, \nu, \gamma, \beta, \delta, \alpha, \mathrm{a}, b),
$$

$\mathrm{H}_{\mathrm{NTAW}}(x, \lambda, \theta, \nu, \gamma, \beta, \delta, \alpha, a, b)=-\ln \left[\left\{1-\left\{(1+\lambda)[I(x, \vartheta)]^{\delta}-\lambda[I(x, \vartheta)]^{\alpha}\right\}^{a}\right\}^{b}\right]$.

\section{Expansion for the PDF and the CDF Functions}

\subsection{Expansion for the pdf function}

Now

$$
(1-z)^{k}=\sum_{j=0}^{\infty} \frac{(-1)^{j} \Gamma(k+1)}{\Gamma(k-j+1) j !} z^{j}
$$

Using (10) and binomial expansion and applying it to (7), the pdf of the Kw-NTAW model can be written as

$$
f(x)=\sum_{i, j, l=0}^{\infty} \sum_{k=0}^{1} A_{i: k}\left(\theta \nu x^{\nu-1}+\gamma \beta x^{\beta-1}\right) e^{-\left(\theta x^{\nu}+\gamma x^{\beta}\right)(l+1)},
$$

where $A_{i: k}$ is a constant term given by

$$
\begin{aligned}
& A_{i: k}= a . b \cdot \frac{(-1)^{i+j+k+l} \Gamma(b) \Gamma(a(i+1))}{i ! l ! j ! \Gamma(b-i) \Gamma(a(i+1)-j)} \lambda^{j+k}(1+\lambda)^{a(i+1)-j-k} \\
& \times \frac{\Gamma(\delta(a(i+1)-1)+\alpha j-\delta j+(\alpha-1) k+(\delta-1)(1-k)-1)}{\Gamma(\delta(a(i+1)-1)+\alpha j-\delta j+(\alpha-1) k+(\delta-1)(1-k)-l-1)} \\
& \text { if } \frac{\lambda[I(x, \vartheta)]^{\alpha}}{(1+\lambda)[I(x, \vartheta)]^{\delta}}<1 \\
& \quad A_{i: k}=a \cdot b \cdot \frac{(-1)^{i+j+k+l} \Gamma(b) \Gamma(a(i+1))}{i ! l ! j ! \Gamma(b-i) \Gamma(a(i+1)-j)}(-\lambda)^{a(i+1)-j-k+1}(1+\lambda)^{j-k+1} \\
& \quad \times \frac{\Gamma(\alpha(\alpha(i+1)-1)-\alpha j+\delta j+(\alpha-1) k+(\delta-1)(1-k)-1)}{\Gamma(\alpha(a(i+1)-1)-\alpha j+\delta j+(\alpha-1) k+(\delta-1)(1-k)-l-1)}
\end{aligned}
$$




\subsection{Expansion for the cdf function}

Using expansion (10) to Equation (6), then the cdf function of the new transmuted additive Weibull distribution can be written as

$$
F(x)=1-\sum_{i, j, k, l=0}^{\infty} B_{i: l}\left(\theta x^{\nu}+\gamma x^{\beta}\right)^{l},
$$

where $B_{i: l}$ is a constant term given by

$$
\begin{gathered}
B_{i: l}=\frac{(-1)^{i+j+k+l} \Gamma(b+1) \Gamma(a(i+1)+1) \Gamma(\delta(a(i+1))-\delta j+\alpha j+1)}{i ! j ! k ! l ! \Gamma(b-i+1) \Gamma(a(i+1)-j+1) \Gamma(\delta(a(i+1))-\delta j+\alpha j-k+1)} \\
\times k^{l} \lambda^{j}(1+\lambda)^{a(i+1)-j}
\end{gathered}
$$

if $\frac{\lambda[I(x, \vartheta)]^{\alpha}}{(1+\lambda)[I(x, \vartheta)]^{\delta}}<1$

$$
\begin{gathered}
B_{i: l}=\frac{(-1)^{i+j+k+l} \Gamma(b+1) \Gamma(a(i+1)+1) \Gamma(\alpha(a(i+1))-\alpha j+\delta j+1)}{i ! j ! k ! l ! \Gamma(b-i+1) \Gamma(a(i+1)-j+1) \Gamma(\alpha(a(i+1))-\alpha j+\delta j-k+1)} \\
\times k^{l}(-\lambda)^{\alpha(i+1)-j}(1+\lambda)^{j} .
\end{gathered}
$$

\section{Statistical Properties}

\subsection{Quantile function}

The quantile function is obtained by inverting the cumulative distribution (12), where the $p$-th quantile $x_{p}$ of Kw-NTAW model is the real solution of the following equation:

$$
1-\sum_{i, j, k, l=0}^{\infty} B_{i: l}\left(\theta x_{p}{ }^{\nu}+\gamma x_{p}{ }^{\beta}\right)^{l}-p=0 .
$$

An expansion for the median $M$ follows by taking $p=0.5$. 


\subsection{Moments}

Theorem 5.1. If $X$ is from a Kw-NTAW distribution, then the $r$-th non-central moments is given by

$$
\mu_{r}^{\prime}=\sum_{i, j, l, m=0}^{\infty} \sum_{k=0}^{1} A_{i: k} \frac{(-1)^{m}(l+1)^{m} \gamma^{m}}{m !}\left[\frac{\theta \nu \Gamma\left(\frac{r+m \beta+\nu}{\nu}\right)}{(\theta(l+1))^{\frac{r+m \beta+\nu}{\nu}}}+\frac{\gamma \beta \Gamma\left(\frac{r+m \beta+\beta}{\nu}\right)}{\left(\theta(l+1) \frac{r+m \beta+\beta}{\nu}\right.}\right],
$$

where $\Gamma($.$) denote the gamma function, i.e.,$

$$
\Gamma(a)=\int_{0}^{\infty} t^{a-1} e^{-t} d t
$$

Proof.

$$
\begin{gathered}
\mu_{r}^{\prime}=E\left(X^{r}\right)=\int_{0}^{\infty} X^{r} f(x, \lambda, \theta, \nu, \gamma, \beta, \delta, \alpha, a, b) d x, \\
\mu_{r}^{\prime}=\int_{0}^{\infty} X^{r}\left\{\sum_{i, j, l=0}^{\infty} \sum_{k=0}^{1} A_{i: k}\left(\theta \nu x^{\nu-1}+\gamma \beta x^{\beta-1}\right) e^{-\left(\theta x^{\nu}+\gamma x^{\beta}\right)(l+1)}\right\} d x, \\
\mu_{r}^{\prime}=\int_{0}^{\infty}\left\{\sum_{i, j, l=0}^{\infty} \sum_{k=0}^{1} A_{i: k}\left(\theta \nu x^{r+\nu-1}+\gamma \beta x^{r+\beta-1}\right) e^{-\left(\theta x^{\nu}+\gamma x^{\beta}\right)(l+1)}\right\} d x, \\
\mu_{r}^{\prime}=\sum_{i, j, l=0}^{\infty} \sum_{k=0}^{1} A_{i: k} I,
\end{gathered}
$$

now, using

$$
\begin{aligned}
& e^{-\left(\gamma x^{\beta}\right)(l+1)}=\sum_{m=0}^{\infty} \frac{(-1)^{m}(l+1)^{m} \gamma^{m} x^{m \beta}}{m !}, \\
& e^{-\left(\theta x^{\nu}\right)(l+1)}=\sum_{m=0}^{\infty} \frac{(-1)^{m}(l+1)^{m} \theta^{m} x^{m \nu}}{m !} .
\end{aligned}
$$


We have

$$
\begin{gathered}
I=\int_{0}^{\infty}\left(\theta \nu x^{r+\nu-1}+\gamma \beta x^{r+\beta-1}\right) e^{-\left(\theta x^{\nu}+\gamma x^{\beta}\right)(l+1)} d x \\
=\int_{0}^{\infty}\left(\theta \nu x^{r+\nu-1}\right) e^{-\left(\theta x^{\nu}\right)(l+1)} e^{-\left(\gamma x^{\beta}\right)(l+1)} d x+\int_{0}^{\infty}\left(\gamma \beta x^{\beta+r-1}\right) e^{-\left(\theta x^{\nu}\right)(l+1)} e^{-\left(\gamma x^{\beta}\right)(l+1)} d x \\
=\sum_{m=0}^{\infty} \frac{(-1)^{m}(l+1)^{m} \gamma^{m}}{m !}\left[\frac{\theta \nu \Gamma\left(\frac{r+m \beta+\nu}{\nu}\right)}{\left(\theta(l+1) \frac{r+m \beta+\nu}{\nu}\right.}+\frac{\gamma \beta \Gamma\left(\frac{r+m \beta+\beta}{\nu}\right)}{(\theta(l+1)) \frac{r+m \beta+\beta}{\nu}}\right]
\end{gathered}
$$

Substituting (15) in (14), we get

$$
\mu_{r}^{\prime}=\sum_{i, j, l, m=0}^{\infty} \sum_{k=0}^{1} A_{i: k} \frac{(-1)^{m}(l+1)^{m} \gamma^{m}}{m !}\left[\frac{\theta \nu \Gamma\left(\frac{r+m \beta+\nu}{\nu}\right)}{(\theta(l+1))^{\frac{r+m \beta+\nu}{\nu}}}+\frac{\gamma \beta \Gamma\left(\frac{r+m \beta+\beta}{\nu}\right)}{(\theta(l+1))^{\frac{r+m \beta+\beta}{\nu}}}\right] .
$$

This completes the proof.

In particular, when $r=1$, Equation (15) yields the mean of the Kw-NTAW distribution, $\mu$, as

$$
\mu=\sum_{i, j, l, m=0}^{\infty} \sum_{k=0}^{1} A_{i: k} \frac{(-1)^{m}(l+1)^{m} \gamma^{m}}{m !}\left[\frac{\theta \nu \Gamma\left(\frac{m \beta+\nu+1}{\nu}\right)}{(\theta(l+1))^{\frac{m \beta+\nu+1}{\nu}}}+\frac{\gamma \beta \Gamma\left(\frac{m \beta+\beta+1}{\nu}\right)}{(\theta(l+1))^{\frac{m \beta+\beta+1}{\nu}}}\right] .
$$

The $n$-th central moments or (moments about the mean) can be obtained easily from the $r$-th non-central moments throw the relation:

$$
m_{u}=E(X-\mu)^{n}=\sum_{r=0}^{n}(-\mu)^{n-r} E\left(X^{r}\right)
$$


Then the $n$-th central moments of the Kw-NTAW is given by

$$
\begin{aligned}
m_{u}= & \sum_{r=0}^{n}(-\mu)^{n-r} \sum_{i, j, l, m=0}^{\infty} \sum_{k=0}^{1} A_{i: k} \frac{(-1)^{m}(l+1)^{m} \gamma^{m}}{m !} \\
& \times\left[\frac{\theta \nu \Gamma\left(\frac{r+m \beta+\nu}{\nu}\right)}{(\theta(l+1))^{\frac{r+m \beta+\nu}{\nu}}}+\frac{\gamma \beta \Gamma\left(\frac{r+m \beta+\beta}{\nu}\right)}{(\theta(l+1))^{\frac{r+m \beta+\beta}{\nu}}}\right] .
\end{aligned}
$$

\section{Simulation Study}

This section explores the behaviours of the proposed estimators in terms of their mean square error on the basis of simulated samples from pdf of Kw-NTAW with varying sample sizes. We take $\lambda=-0.55, \theta=3$, $\nu=0.8, \gamma=2, \beta=2.5, a=2, b=4, \delta=3$, and $\alpha=2$ arbitrarily and $n=10(10) 100$. The algorithms are coded in $\mathrm{R}$ has been used for simulation purposes. We calculate MLE estimators of $\lambda, \theta, \nu, \gamma, \beta, \delta, \alpha, a$, and $b$ based on each generated sample. This simulation is repeated 1000 of times, and average estimates with corresponding mean square errors are computed and reported in Table 2 . 
Table 2. Estimates and mean square errors (in 2-nd row of each cell) of the proposed estimators with varying sample size

\begin{tabular}{|c|c|c|c|c|c|c|c|c|c|}
\hline \multirow{2}{*}{$n$} & \multicolumn{9}{|c|}{ MLE } \\
\hline & $\lambda$ & $\theta$ & $v$ & $\gamma$ & $\beta$ & $\delta$ & $\alpha$ & $a$ & $b$ \\
\hline \multirow{2}{*}{10} & -0.5524 & 3.6501 & 0.7034 & 2.6431 & 2.5641 & 3.0175 & 2.5544 & 2.0652 & 3.8801 \\
\hline & 0.1270 & 1.9205 & 0.2261 & 1.8215 & 0.9885 & 0.1300 & 1.2817 & 0.0885 & 1.9961 \\
\hline \multirow{2}{*}{20} & -0.5558 & 3.4466 & 0.6958 & 2.5516 & 2.4454 & 3.0022 & 2.2391 & 2.0622 & 3.8455 \\
\hline & 0.0489 & 1.4783 & 0.1485 & 1.7723 & 0.9283 & 0.0507 & 0.3825 & 0.0722 & 0.4558 \\
\hline \multirow{2}{*}{30} & 0.5406 & 2.9786 & 0.7006 & 2.7786 & 2.2786 & 3.9949 & 2.1573 & 2.0381 & 3.8336 \\
\hline & 0.0299 & 0.4971 & 0.222 & 1.4871 & 0.8972 & 0.0307 & 0.2298 & 0.0364 & 0.2553 \\
\hline \multirow{2}{*}{40} & -0.5634 & 2.8352 & 0.6637 & 2.7352 & 2.1362 & 3.9952 & 2.1215 & 2.0146 & 3.8654 \\
\hline & 0.0253 & 0.3510 & 0.2103 & 0.8520 & 0.7510 & 0.0224 & 0.1590 & 0.0312 & 0.2419 \\
\hline \multirow{2}{*}{50} & -0.6013 & 2.7542 & 0.6803 & 2.7002 & 2.0042 & 3.9954 & 2.0965 & 2.0203 & 3.8742 \\
\hline & 0.0181 & 0.2685 & 0.2172 & 0.5445 & 0.7285 & 0.0184 & 0.1252 & 0.0269 & 0.1802 \\
\hline \multirow{2}{*}{60} & -0.6101 & 2.8154 & 0.6101 & 2.6654 & 1.8154 & 3.9956 & 2.0804 & 2.0106 & 3.9612 \\
\hline & 0.0168 & 0.2379 & 0.1608 & 0.5229 & 0.2379 & 0.0148 & 0.0998 & 0.0187 & 0.1379 \\
\hline \multirow{2}{*}{70} & -0.6410 & 2.7223 & 0.6010 & 2.2211 & 1.7273 & 3.9966 & 2.0711 & 3.0101 & 3.9700 \\
\hline & 0.0119 & 0.2175 & 0.1519 & 0.2005 & 0.2175 & 3.0125 & 0.0872 & 0.0112 & 0.1175 \\
\hline \multirow{2}{*}{80} & -0.6627 & 2.7700 & 0.6007 & 2.5510 & 1.7640 & 3.9978 & 2.0553 & 2.0100 & 3.9932 \\
\hline & 0.0100 & 0.0109 & 0.1400 & 0.0228 & 0.1007 & 4.0106 & 0.0671 & 0.0132 & 0.1089 \\
\hline \multirow{2}{*}{90} & -0.6688 & 2.6920 & 0.5988 & 2.5220 & 1.6920 & 3.9992 & 2.0511 & 2.0104 & 3.9943 \\
\hline & 0.0089 & 0.0089 & 0.1089 & 0.0879 & 0.0801 & 3.0095 & 0.0619 & 0.0073 & 0.0784 \\
\hline \multirow{2}{*}{100} & -0.6993 & 2.6518 & 0.5803 & 2.5524 & 1.0517 & 3.9982 & 2.0471 & 2.0087 & 3.9938 \\
\hline & 0.0073 & 0.0085 & 0.1062 & 0.0074 & 0.0099 & 0.0087 & 0.0545 & 0.0082 & 0.0675 \\
\hline
\end{tabular}

From Table 2, it can be clearly observed that as sample size increases the mean square error decreases, which proves the consistency of the estimators.

\section{Application}

In this section, we use a real data set to see how the new model works in practice. Compare the fits of the Kw-NTAW distribution with others models. In each case, the parameters are estimated by maximum likelihood, using the $\mathrm{R}$ code. 
In order to compare the two distribution models, we consider criteria like $-2 \mathcal{L}$, $\mathrm{AIC}$ (Akaike information criterion), $\mathrm{AIC}_{C}$ (corrected Akaike information criterion), and BIC (Bayesian information criterion) for the data set. The better distribution corresponds to smaller $-2 \mathcal{L}$, AIC, and $\mathrm{AIC}_{C}$ values:

$$
\begin{gathered}
\mathrm{AIC}=-2 \mathcal{L}+2 k, \\
\mathrm{AIC}_{C}=-2 \mathcal{L}+\left(\frac{2 k n}{n-k-1}\right),
\end{gathered}
$$

and

$$
\mathrm{BIC}=-2 \mathcal{L}+k \log (n)
$$

where $\mathcal{L}$ denotes the log-likelihood function evaluated at the maximum likelihood estimates, $k$ is the number of parameters, and $n$ is the sample size.

The data set represents failure time of 50 items reported in Aarset [1]. Some summary statistics for the failure time data are as follows:

$\begin{array}{cccccc}\text { Min. } & \mathbf{1}^{\text {st }} \mathbf{Q u .} & \text { Median } & \text { Mean } & \mathbf{3}^{\text {rd }} \mathbf{Q u .} & \text { Max. } \\ 0.10 & 13.50 & 48.50 & 45.67 & 81.25 & 86.00\end{array}$


Table 3. MLEs the measures $\mathrm{AIC}, \mathrm{AIC}_{C}$, and BICS test to failure time data for the models

\begin{tabular}{|c|c|c|c|c|c|}
\hline Model & Parameter estimates & $-\log L$ & AIC & $\mathrm{AIC}_{C}$ & BIC \\
\hline \multirow{9}{*}{ KNTAW } & $\lambda=-0.276021$ & \multirow{9}{*}{199.227} & \multirow{9}{*}{453.3686} & \multirow{9}{*}{457.8686} & \multirow{9}{*}{433.6628} \\
\hline & $\theta=0.002148$ & & & & \\
\hline & $v=2.1528400$ & & & & \\
\hline & $\gamma=0.0592109$ & & & & \\
\hline & $\beta=0.433020$ & & & & \\
\hline & $\delta=0.306828$ & & & & \\
\hline & $a=0.0535599$ & & & & \\
\hline & $a=0.22353$ & & & & \\
\hline & $b=0.051958$ & & & & \\
\hline \multirow{5}{*}{ TAW } & $\lambda=0.0076999983$ & \multirow{5}{*}{229.3821} & \multirow{5}{*}{468.7642} & \multirow{5}{*}{470.1278} & \multirow{5}{*}{478.3243} \\
\hline & $\theta=0.00010750$ & & & & \\
\hline & $\nu=2.1463000$ & & & & \\
\hline & $\gamma=0.083400002$ & & & & \\
\hline & $\beta=0.4152000011$ & & & & \\
\hline \multirow{5}{*}{ TEMW } & $\lambda=-0.1640672$ & \multirow{5}{*}{236.6535} & \multirow{5}{*}{487.6286} & \multirow{5}{*}{488.992} & \multirow{5}{*}{497.1887} \\
\hline & $\theta=0.0176781$ & & & & \\
\hline & $\boldsymbol{y}=\mathbf{0 . 0 0 1 9 3 2 9 8}$ & & & & \\
\hline & $\beta=0.03926070$ & & & & \\
\hline & $\alpha=0.949241462$ & & & & \\
\hline \multirow{4}{*}{ EMW } & $\theta=0.018673571$ & \multirow{4}{*}{238.8143} & \multirow{4}{*}{481.307} & \multirow{4}{*}{482.1959} & \multirow{4}{*}{488.9551} \\
\hline & $\gamma=0.001822666$ & & & & \\
\hline & $\beta=0.010505798$ & & & & \\
\hline & $\alpha=0.703411609$ & & & & \\
\hline
\end{tabular}


Table 3. (Continued)

\begin{tabular}{|c|c|c|c|c|c|}
\hline \multirow{4}{*}{ AW } & $\theta=0.0002399$ & \multirow{4}{*}{237.7583} & \multirow{4}{*}{483.5166} & \multirow{4}{*}{484.4055} & \multirow{4}{*}{491.1647} \\
\hline & $\nu=1.852800617$ & & & & \\
\hline & $\gamma=0.016255755$ & & & & \\
\hline & $\beta=0.9475073247$ & & & & \\
\hline \multirow{3}{*}{ MW } & $\theta=1.827194$ & \multirow{3}{*}{241.0289} & \multirow{3}{*}{488.0578} & \multirow{3}{*}{488.5795} & \multirow{3}{*}{493.7939} \\
\hline & $\gamma=1.80309$ & & & & \\
\hline & $\beta=1.000288$ & & & & \\
\hline \multirow{2}{*}{$\mathbf{W}$} & $\gamma=0.9489561$ & \multirow{2}{*}{240.9796} & \multirow{2}{*}{485.959} & \multirow{2}{*}{486.2145} & \multirow{2}{*}{489.7832} \\
\hline & $\beta=0.02227559$ & & & & \\
\hline \multirow{3}{*}{ TEE } & $\widehat{\lambda}=0.84 * 10^{-5}$ & \multirow{3}{*}{238.6896} & \multirow{3}{*}{483.3793} & \multirow{3}{*}{483.9011} & \multirow{3}{*}{489.1154} \\
\hline & $\widehat{\beta}=1.69 * 10^{-4}$ & & & & \\
\hline & $\widehat{a}=0.30966$ & & & & \\
\hline \multirow{3}{*}{ EE } & $\widehat{p}=0.09240$ & \multirow{3}{*}{239.9733} & \multirow{3}{*}{483.9467} & \multirow{3}{*}{484.2021} & \multirow{3}{*}{487.7708} \\
\hline & $\widehat{\lambda}=-3.21 * 10^{-5}$ & & & & \\
\hline & $\hat{a}=2.33 * 10^{-3}$ & & & & \\
\hline \multirow{2}{*}{ LE } & $\widehat{\lambda}=0.01364528$ & \multirow{2}{*}{238.048836} & \multirow{2}{*}{480.0977} & \multirow{2}{*}{480.353} & \multirow{2}{*}{483.9217} \\
\hline & $\widehat{\beta}=0.00023990$ & & & & \\
\hline $\mathbf{E}$ & $\widehat{\beta}=0.02189828$ & 241.0677 & 484.1354 & 484.2187 & 486.0474 \\
\hline
\end{tabular}

These results indicate that the Kw-NTAW model has the lowest AIC and $\mathrm{AIC}_{C}$ and BIC values among the fitted models. The values of these statistics indicate that the Kw-NTAW model provides the best fit to this data. 


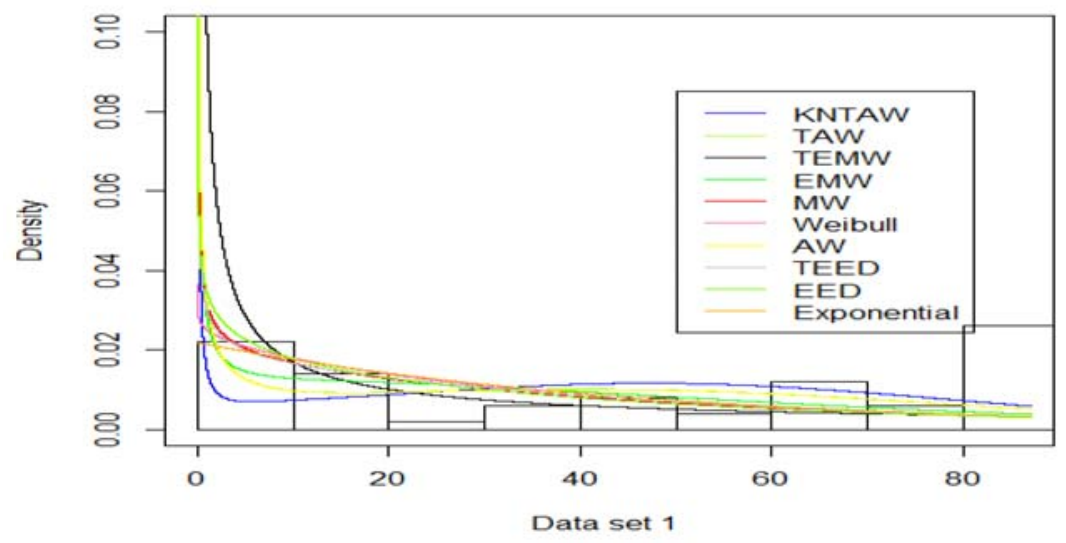

Figure 3. Estimated densities of the data set.

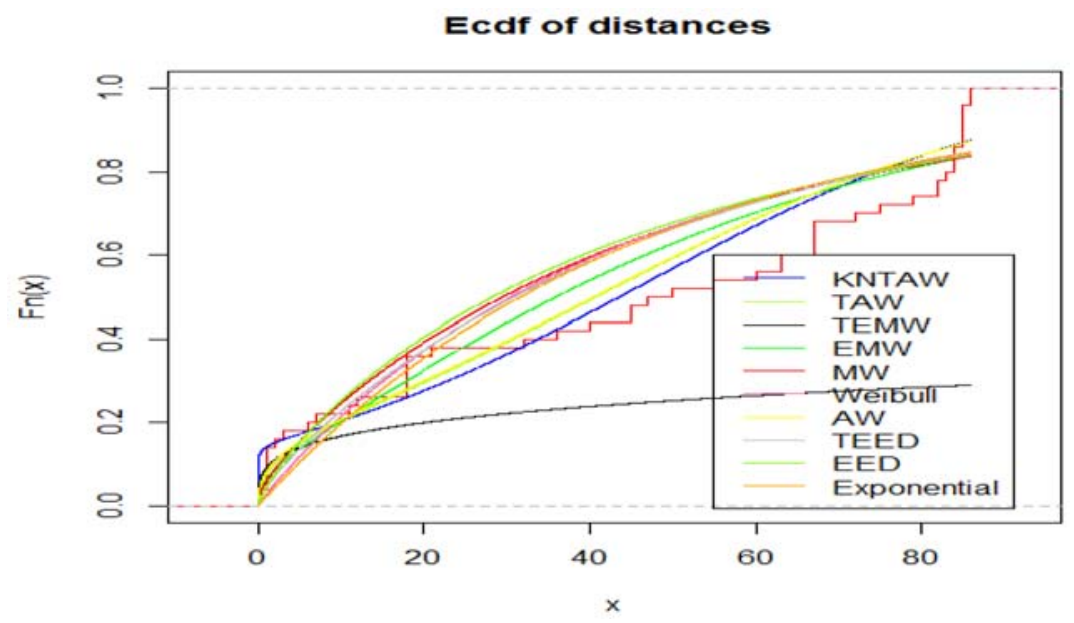

Figure 4. Empirical, fitted, Kw-NTAW, TAW, TEMW, EMW, MW, Weibull, AW, TEE, EE, and exponential distributions of the data set.

\section{References}

[1] M. V. Aarset, How to identify a bathtub hazard rate, IEEE Transactions on Reliability 36(1) (1987), 106-108.

[2] G. M. Cordeiro and M. de Castro, A new family of generalized distributions, Journal of Statistical Computation and Simulation 81(7) (2011), 883-898. 
[3] I. Elbatal and G. Aryal, On the transmuted additive Weibull distribution, Austrian Journal of Statistics 42(2) (2013), 117-132.

[4] R. C. Gupta, P. L. Gupta and R. D. Gupta, Modeling failure time data by Lehman alternatives, Communications in Statistics-Theory and Methods 27(4) (1998), 887-904.

[5] R. Jiang, P. Ji and X. Xiao, Ageing property of unimodal failure rate models, Reliability Engineering \& System Safety 79(1) (2003), 113-116.

[6] P. Kumaraswamy, A generalized probability density function for double-bounded random processes, Journal of Hydrology 46(1) (1980), 79-88.

[7] M. M. Mansour and S. M. Hamed, A new generalization of power Lindley distribution: With applications to lifetime data, Journal of Statistics: Advances in Theory and Applications 13(1) (2015), 33-65.

[8] G. S. Mudholkar, D. K. Srivastava and M. Freimer, The exponentiated Weibull family: A reanalysis of the bus-motor-failure data, Technometrics 37(4) (1995), 436-445.

[9] T. Zhang, M. Xie, L. C. Tang and S. H. Ng, Reliability and modeling of systems integrated with firmware and hardware, International Journal of Reliability, Quality and Safety Engineering 12(03) (2005), 227-239. 